TAO, Vol. 16, No. 3, 677-689, August 2005

\title{
CISK Instability of Equatorial Kelvin Waves Using the Evaporation-Wind Feedback Mechanism as the Cumulus Parameterization Scheme
}

\author{
Han-Ru Cho ${ }^{1, *}$, Pay-Liam Lin ${ }^{1}$ and Jough-Tai Wang ${ }^{1}$ \\ (Manuscript received 5 January 2004, in final form 16 June 2005)
}

\begin{abstract}
The instability of the Kelvin waves problem is analyzed using the evaporation-wind feedback mechanism as the cumulus parameterization scheme. The method we used is a modified method of characteristics (LeVeque 1992).

Two cases were considered. First was linear case without assuming any background wind. We found that at lower levels the perturbation will be unstable only if the wind is westerly. At the upper troposphere, in order to be unstable the wind must be easterly, and the system is stable when the perturbation wind is westerly. Next, we calculated the linear case with a background wind in the zonal direction. This is identical to the case analyzed by Neelin et al. (1987). We also obtained identical results, namely the system is stable if the background wind is assumed westerly and unstable if the background wind is assumed easterly.
\end{abstract}

(Key words: CISK instability, Kelvin wave, Evaporation-Wind feedback mechanism, Cumulus parameterization )

\section{INTRODUCTION}

Recently Cho (2000a, b) has placed a question mark on the validity of the concept of Kelvin-wave-CISK. But in his argument, he has used a particular form of a cumulus parameterization scheme. In the conclusions of these papers the author stated that in order to prove this conclusion in general, there is the need to carry out the proof using several different cumulus parameterization schemes, at least for those schemes that are easily tractable by analytic

\footnotetext{
${ }^{1}$ Institute of Atmospheric Physics, National Central University, Chung-Li, Taiwan, ROC

* Corresponding author address: Prof. Han-Ru Cho, Institute of Atmospheric Physics, National Central University, Chung-Li, Taiwan, ROC;E-mail: cho@atm.ncu.edu.tw
} 
means.

There seems to be two very different interpretations of the concept of CISK in general Kelvin wave-CISK in particular. One widespread interpretation is that CISK is a cooperative interaction between the low-level convergence produced by a meso-beta or larger scale disturbance and the conditional unstable atmosphere where the disturbance is located in. The lowlevel convergence produces the initial ascent needed to trigger convection. The convective heating then feeds back onto the disturbance.

The other interpretation, although not as popular as the previous one, has its own firm believers. According to this interpretation, CISK is a cooperative phenomenon between disturbances at two different scales, one being cumulus convection at the meso-gamma scale, the other being a larger scale disturbance. While the cumulus convection (meso-gamma scale) supplies energy to the larger scale disturbance through its latent heat release, the larger scale disturbance helps maintain the cumulus convection by supplying the moisture which is used as fuel by cumulus convection. The details of the interaction is left to the cumulus parameterization scheme one adopts; for instance, whether it be a low level convergence or evaporation from the ocean surface. In a GCM, for example, if adopting the Arakawa and Schubert scheme, then it would still be appropriate to call any larger scale disturbance induced by the cumulus scheme a CISK phenomenon.

In this paper we shall adopt this second viewpoint. The purpose of this paper is to present the discussions of the existence or the nonexistence of instability of Equatorial Kelvin waves using another parameterization scheme other than that of low level convergence, namely, the wind-evaporation feedback scheme as proposed by Emanuel (1987) and Neelin et al. (1987).

The organization of the paper is as follows: a discussion of the wind-evaporation feedback scheme can be found in section 2. A modified method of characteristics that will be used in this paper is found in section 3. It is then followed by a discussion on the existence or nonexistence of instability of Kelvin waves in a background atmosphere with zero wind speed. In section 4, we will consider a perturbation in a background atmosphere with a mean zonal wind. Concluding remarks are made in section 5.

\section{WIND-EVAPORATION FEEDBACK SCHEME}

The scheme assumes that variation in the amount of cumulus clouds is proportional to the evaporation rate from the surface. Since the surface evaporation rate is proportional to surface wind speed therefore, according to this theory, the heating rate by cumulus clouds can be written as (Neelin et al. 1987):

$$
Q=A\left|u_{s}\right| f(p),
$$

A is a positive constant so that $\mathrm{Q}$ is a positive whenever $\mathrm{f}(\mathrm{p})$ is positive. $u_{s}$ is the surface wind on the ocean and $f(p)$ gives vertical distribution of $Q$. We shall adopt the convention that $f(p)$ is normalized in the sense that: 


$$
\frac{1}{\Delta p} \int_{p_{t}}^{p_{s}} f(p) d p=1
$$

where $p_{s}$ and $p_{t}$ the pressures at surface and the tropopause levels, respectively and $\Delta p=p_{s}-p_{t}$.

\section{METHOD OF CHARACTERISTIC}

The method of characteristic works only for hyperbolic equations (i.e., no dispersive waves). It is designed for initial value problems. It works equally well for dispersive waves provided that the dispersive terms (if one is able to separate them out) are only responsible for the exponential growth or decay of the wave.

The method's main advantage is that it treats nonlinear problems in exactly the same way as linear problems. Only the algebra very often is impossibly complicated when one is treating a nonlinear problem. When applied to instability analysis the stability is more transparent because the method follows the physical reasoning very closely (in contrast to normal mode method where everything is lumped into the dispersion relation). While applying the normal mode method many scientists have found the Kelvin-wave and cumulus heating problem (CISK) unstable (see Cho and Wang 2001), while applying the method of characteristics Cho (2000a) was able to show the Kelvin waves were actually stable. In this section, we shall assume that there is no east-west background wind and a constant background geopotential height. If the linear approximation is made, and assume that $\mathrm{U}$ and $\phi$ are the $\mathrm{t}$ - $\mathrm{x}$ varying component of the zonal wind $\mathrm{u}$ and the geopotential $\Phi=g z$, then the system is governed by:

$$
\begin{aligned}
& \frac{\partial u}{\partial t}+\frac{\partial \Phi}{\partial x}=0, \\
& \frac{\partial}{\partial t}\left(\frac{\partial \Phi}{\partial p}\right)+\sigma \omega=-Q, \\
& \frac{\partial \Phi}{\partial y}+\beta y u=0, \\
& \frac{\partial u}{\partial x}+\frac{\partial \omega}{\partial p}=0, \\
& \frac{\partial \Phi}{\partial p}=-\frac{R T}{g} .
\end{aligned}
$$


These equations, in the order they appear, are the east-west momentum equation, the thermodynamic equation, geostrophic balance in the $y$-direction, mass continuity equation, and the gravity balance equation, respectively.

From (2a) and (2d) we see that the vertical variations of the following two quantities are given by $h^{\prime}(p)$ where prime means taking the derivative:

$$
\begin{aligned}
& u(x, t, p, y)=U(x, t) U_{0}(y) h^{\prime}(p) \\
& \Phi(x, t, p, y)=\phi(x, t) \phi_{0}(y) h^{\prime}(p),
\end{aligned}
$$

$\mathrm{h}(\mathrm{p})$ is the vertical profile of $\omega$, and it can be assumed to be a positive quantity. From (2b) we get:

$$
\begin{aligned}
& \frac{\partial}{\partial t}\left(\frac{\partial^{2} \Phi}{\partial p^{2}}\right)-\sigma \frac{\partial u}{\partial x}=-\frac{\partial Q}{\partial p}, \text { or } \\
& \frac{\partial \phi}{\partial t}-\frac{\sigma h^{\prime}(p)}{h^{\prime \prime \prime}(p)} \frac{\partial U}{\partial x}=-\frac{\partial U}{\partial p} \frac{1}{h^{\prime \prime \prime}(p)},
\end{aligned}
$$

if we let $\Psi=\left\{\begin{array}{l}U \\ \phi\end{array}\right\}$, then the prognostic equations become:

$$
\frac{\partial \Psi}{\partial t}+B \frac{\partial \Psi}{\partial x}=\left\{-\frac{\partial Q}{\partial p} \frac{0}{h^{\prime \prime \prime}(p)}\right\}=\Gamma
$$

where $\mathrm{B}$ is the matrix:

$$
\left(\begin{array}{cc}
0 & 1 \\
-\sigma \frac{h^{\prime}(p)}{h^{\prime \prime \prime}(p)} & 0
\end{array}\right) .
$$

Since we are studying waves, we can introduce a factor c such that:

$$
-c I \frac{\partial \Psi}{\partial x}=\frac{\partial \Psi}{\partial t}
$$

where I is identity matrix and $\mathrm{c}$ is the propagation speed of a Kelvin wave. We can always do this for all wave-like phenomenon including exponentially growing or decaying waves. Then (5) becomes: 


$$
(c I-B) \frac{\partial \Psi}{\partial x}=-\Gamma
$$

The eigenvalues of the matrix B have the same dimension as a speed. Actually, we will prove a posteriori that the right hand term of (7b) will only make the solution grow or decay exponentially. Therefore, the eigenvalues of the matrix B will provide the propagation speed of the Kelvin wave (LeVeque 1992). The propagation speeds of the Kelvin waves are therefore:

$$
\lambda_{1,2}= \pm \sqrt{-\sigma \frac{h^{\prime}(p)}{h^{\prime \prime \prime}(p)}},
$$

if $\sigma \frac{h^{\prime}(p)}{h^{\prime \prime \prime}(p)}$ is a negative quantity. Since Kelvin waves are eastward propagating only, the positive solution is corresponding to the "real" solution. Furthermore, if Kelvin waves propagating at various levels are with the same propagation speed, they should be the same wave, and then $\lambda_{1,2}$ should not be a function of p. From (2a) we obtain:

$$
\frac{d x}{d t} d U=-d \phi
$$

or by integration:

$$
c U=-\phi,
$$

$c$ is a positive number because we know that the propagation velocity is toward the east for Kelvin waves. Substituting this into the vertical derivative of the thermodynamic equation we get:

$$
\frac{\partial\left(-c U h^{\prime \prime \prime}\right)}{\partial t}-\sigma \frac{\partial U}{\partial x} h^{\prime}=-\frac{\partial Q}{\partial p}
$$

This equation says that along:

$$
\begin{aligned}
& \frac{d x}{d t}=\frac{\sigma h^{\prime}(p)}{c h^{\prime \prime \prime}(p)}, \\
& \frac{d U}{d t}=\left\{\partial\left[A\left|u_{s}\right| f(p)\right] / \partial p\right\} /\left[c h^{\prime \prime \prime}(p)\right] .
\end{aligned}
$$


This is the central spirit of the method of characteristics. The statement "along $\mathrm{dx} / \mathrm{dt}=\sigma \mathrm{h}^{\prime} / \mathrm{ch}^{\prime \prime}$ "gives the line of characteristics. If the right side of (10c) is zero then we have the pure method of characteristics. If the right side of (10c) is not equal to zero, but equal to something that is proportional to $U$ then we have the modified method of characteristics.

Note that we have $h^{\prime}(p) / h^{\prime \prime}(p)=h(p) / h^{\prime \prime}(p)$ (Goldstein 1980, see appendix II) $h(p)$ is usually convex downward with respect to $p$ axis (that is, the negative of vertical velocity function $-\omega$ has a maximum somewhere in the middle troposphere) therefore $h "(p)$ is a negative quantity in the cloud regions. Assuming that:

$$
\left|u_{s}\right|=\left|U(x, t) U_{0}(y)\right|\left|h^{\prime}(p=0)\right|=\alpha(p) u(p),
$$

where $\alpha$ could be either positive or negative depending on the sign of $\mathrm{u}$. The middle part of the equality is based on (3). Substitution of this relation into (10c), making use of (3), gives:

$$
\begin{aligned}
& \frac{d U}{d t}=A \alpha(p) u(p) f^{\prime}(p) \frac{1}{c h^{\prime \prime \prime}(p)}=-E \alpha U, \\
& E=-A U_{0}(y) f^{\prime}(p) \frac{h^{\prime}(p)}{c h^{\prime \prime \prime}(p)}=-A U_{0}(y) f^{\prime}(p) \frac{h(p)}{c h^{\prime \prime}(p)} \\
& =A U_{0}(y) \frac{f^{\prime}(p)}{c}\left|\frac{h(p)}{h^{\prime \prime}(p)}\right| .
\end{aligned}
$$

Then we get $U(t)=U(0) \exp (-E \alpha U)$. If $\mathrm{E}>0$ and the perturbation wind is westerly, i.e., then $U$ will decrease in time exponentially. Therefore it is stable. On the other hand, if then it is unstable. Thus the stability will depend not only on the sign of $u$, but also depends on the sign of $\mathrm{E}$ (thereby being dependent on the sign of $\mathrm{f}^{\prime}$ ). At lower levels the heating is most likely an increasing function of height or a decreasing function of pressure. At lower levels then $\mathrm{E}<0$, u must be a westerly to be unstable (the westerly wind burst?). At upper troposphere, all the signs are reversed: E most likely to be positive. In order to be unstable the wind should be easterly.

\section{SMALL AMPLITUDE ANALYSIS OF THE CASE WITH A CONSTANT BACK- GROUND ZONAL WIND}

This case has been analyzed by Neelin et al. (1987). Since our method is somewhat different, we have singled out this case for comparisons.

The equation corresponding to (9) can be written in this case:

$$
\phi=\left(c-u_{0}\right) U
$$


Substitution into the thermodynamic equation of this we obtain:

$$
\frac{\partial U}{\partial t}+u_{0} \frac{\partial U}{\partial x}-\frac{\sigma}{c-u_{0}} \frac{h(p)}{h^{\prime \prime}(p)} \frac{\partial U}{\partial x}=-\frac{\partial Q / \partial p}{\left(c-u_{0}\right) h^{\prime \prime \prime}(p)}
$$

We have assumed that $c-u_{0} \neq 0$ and divided the entire equation by it. The equation says along the characteristics line:

$$
\begin{aligned}
& \frac{d x}{d t}=u_{0}-\sigma \frac{h(p)}{\left(c-u_{0}\right) h^{\prime \prime}(p)}, \\
& \frac{d U}{d t}=-\frac{\partial Q / \partial p}{\left(c-u_{0}\right) h^{\prime \prime \prime}(p)},
\end{aligned}
$$

or

$$
\begin{aligned}
\frac{d U(x, t)}{d t} & =+\frac{A\left|u_{s}\right| \frac{\partial f(p)}{\partial p}}{(c-u) h^{\prime \prime \prime} f(p)} \\
& =\frac{A\left|u_{s}\right| f^{\prime}(p)}{\left(c-u_{0}\right) h^{\prime \prime \prime}(p)} \\
& =-\frac{A \alpha(p) f^{\prime}(p) h(p) U_{0}(y)}{\left(c-u_{0}\right)\left|h^{\prime \prime}(p)\right|} U(x, t) .
\end{aligned}
$$

The last part of the equality again is based on (3) and on the equality also an $\alpha$ which intends to link surface wind speed to those at other height levels. The sign convention is the same as before. If $-u_{0}>0$ then $\alpha f^{\prime}<0$ to be unstable. Usually $\mathrm{h}(\mathrm{p})$ and $\mathrm{U}_{0}$ are both positive. Despite $\mathrm{U}$ being infinitesimal, $\alpha$ is positive if $\mathrm{U}$ is positive and vice versa. We have already explained before, this is most likely to happen in the lower troposphere. On other hand if negative $\mathrm{U}$ is to be exponentially unstable, we have $f^{\prime}(p)>0$. This is most likely to happen in the upper troposphere. If $c-u_{0}<0$ then $\alpha f^{\prime}>0$ is unstable. $c$ is always positive, in order that $c-u_{0}<0, \mathrm{c}<u_{0}$. There are many other conditions for the system to be unstable, but to enumerate them would be a rather boring exercise. Neelin et al.'s (1987) case used a shallow water system that has contributed much to their simpler conditions for instability. Our added dimension (in the vertical direction) really complicated the analysis so much so we don't think a simple comparison is possible. The most we can say is their results seem to be consistent with ours. 


\section{CONCLUSIONS}

We have used the method of characteristics to determine the CISK instability characteristics of Kelvin waves using the evaporation-wind feedback scheme to parameterize cumulus clouds. But the method is applicable to only non-dispersive waves when cumulus heating is absent. When cumulus heating is present the method is applicable if cumulus heating only contributes to the exponential growth or decay of the wave.

In infinitesimal analysis without any background wind we found that stability depends not only on the direction of perturbation wind, but also on vertical heating distribution. At lower levels the heating is most likely a decreasing function of pressure, and the perturbation will be unstable only if the wind is westerly.

At the upper troposphere, all the signs are reversed: In order to be unstable the wind must be easterly. At present we have no way of knowing what is responsible for the east-west symmetry breaking. As to an explanation for the phenomena of westerly wind burst, we can only say these are puzzles we cannot explain.

Because we used an entirely different method than the usual normal mode perturbation technique, we also repeated a case did by Neelin et al. (1987), namely a normal mode analysis assuming an infinitesimal perturbation, but superimposed on a uniform background zonal wind $u_{0}$, except that Neelin et al.'s analysis is confined to a shallow water system with a strong damping factor, while our model is based on primitive equations. No simple comparisons of the results from these two models are possible. The best we can say is that the two sets of models are consistent with one another.

\section{REFERENCES}

Cho, H. R., 2000a: Nonlinear Equatorial Kelvin waves and CISK. Part I: Small Amplitude approximation and the Trailing edge of a cloud region. Quart. J. Roy. Meteor. Soc., 126, $569-579$.

Cho, H. R., 2000b: Nonlinear Equatorial Kelvin waves and CISK. Part II: Finite amplitude analysis and the leading edge of a cloud region. Quart. .J. Roy. Meteor. Soc., 126, 58058.

Li, C. Y., H. R. Cho, and J. T. Wang, 2002: CISK Kelvin wave with evaporation-wind feedback and air-sea interaction - A further study of tropical intraseasonal oscillation mechanism. Adv. Atmos., 19, 379-390.

Emanuel, K., 1987: An air-sea interaction model of intraseasonal oscillations in the tropics.J. Atmos. Sci., 44, 2324-2340.

Goldstein, H., 1982: Classical Mechanics, 2nd Edition, John-Wesley.

LeVeque, R. J. 1992: Numerical methods for conservation laws. Birkhauser Verlag, Basel, Swizerland.

Neelin, J. D., I. M. Held, and K. H. Cook, 1987: Evaporation-Wind feedback and the low frequency variability in the tropical atmosphere.J. Atmos. Sci., 44, 2341-2348. 
Lau, K. M., and C. H. Sui, 1997: Mechanisms of short-term sea surface temperature regulation: observation during TOGA COARE. J. Climate, 10, 465-472.

Neelin, J. D., and J. Y. Yu, 1994: Modes of tropical variability under convective adjustment and the Madden-Julian Oscillation. Part I: Analytical theory.J. Atmos. Sci., 51, 1876-1894.

Yu, J. Y., and J. D. Neelin, 1994: Modes of tropical variability under convective adjustment and the Madden-Julian Oscillation. Part II: Numerical results. J. Atmos. Sci., 51, 18951914.

\section{Appendix I}

\section{A Proof that the}

\section{Kelvin Waves here are Dispersive Waves}

The governing equations of equatorial Kelvin waves are:

$$
\begin{aligned}
& \frac{\partial u}{\partial t}+\frac{\partial \Phi}{\partial x}=0, \\
& \frac{\partial}{\partial t}\left(\frac{\partial \Phi}{\partial p}\right)+\sigma \omega=-Q \\
& \frac{\partial \Phi}{\partial y}+\beta y u=0, \\
& \frac{\partial u}{\partial x}+\frac{\partial \omega}{\partial p}=0 \\
& \frac{\partial \Phi}{\partial p}=-\frac{R T}{g}
\end{aligned}
$$

We want to prove that a Kelvin wave, with no background zonal wind, supported by a wind-evaporation feedback scheme is a dispersive wave.

Assume a normal mode structure:

$$
\operatorname{Exp}[\mathrm{ikx}-\mathrm{i} \Omega \mathrm{t}]
$$

Substituting it into the governing equations we obtain: 


$$
\begin{aligned}
& -i \Omega u+i k \Phi=0 \\
& -i \Omega\left(\frac{\partial \Phi}{\partial p}\right)+\sigma \omega=-Q \\
& \frac{\partial \Phi}{\partial y}+\beta y u=0 \\
& i k u+\frac{\partial \omega}{\partial p}=0 \\
& \frac{\partial \Phi}{\partial p}=-\frac{R T}{g}
\end{aligned}
$$

As before define $h(p)$ to be the vertical profile of $\omega$. Then from (I-2a) and (I-2d) we obtain that the vertical profile for $\mathrm{u}$ and $\Phi$ are $\mathrm{h}^{\prime}(\mathrm{p})$. Taking $\mathrm{p}$-derivative of (I-2b) to get:

$$
-\mathrm{i} \Omega \frac{\partial^{2} \Phi}{\partial p^{2}}+\sigma \frac{\partial \omega}{\partial p}=-\frac{\partial Q}{\partial p}
$$

Taking derivative twice with respect to $\mathrm{p}$ of (I-2a) we get:

$$
-\mathrm{i} \Omega \frac{\partial^{2} u}{\partial p^{2}}+i k \frac{\partial^{2} \Phi}{\partial p^{2}}=0
$$

Thus

$$
+\mathrm{i} \frac{\Omega^{2}}{k} \frac{\partial^{2} u}{\partial p^{2}}+i k \sigma u=+A\left|u_{s}\right| f^{\prime}(p)
$$

Let $\left|u_{s}\right|=g(p) u_{h}$ where the sign of $g(p)$ is the same as that of $u_{h}$. Then we have:

$$
\mathrm{i} \frac{\Omega^{2}}{k} u_{h} h^{\prime \prime \prime}(p)+i k \sigma u_{h} h^{\prime}(p)=A u_{h} g(p) f^{\prime}(p)
$$

where subscript $\mathrm{h}$ means that the function is a function of $\mathrm{x}, \mathrm{y}, \mathrm{t}$ only. With little reflection, it will become clear that $\mathrm{g}(\mathrm{p})=\operatorname{sign}[\mathrm{g}(\mathrm{p})]\left|\mathrm{h}^{\prime}(\mathrm{p})\right|=\operatorname{sign}\left(u_{h}\right)\left|\mathrm{h}^{\prime}(\mathrm{p})\right|$. We divide (I-4) by the function 
$\mathrm{h}^{\prime \prime}(\mathrm{p})$ as well as $u_{h}$ :

$$
\mathrm{i} \frac{\Omega^{2}}{k}+i k \frac{h^{\prime}(p)}{h^{\prime \prime \prime}(p)}=A \frac{g(p) f^{\prime}(p)}{h^{\prime \prime \prime}(p)}
$$

from $(7 \mathrm{c})$ we have:

$$
-\frac{h^{\prime}(p)}{h^{\prime \prime \prime}(p)}=\frac{c^{2}}{\sigma}
$$

where $\mathrm{c}$ is the propagation speed of Kelvin waves which should not be a function of height (pressure). We have assumed that $\sigma$ is independent of height. Since the left hand side of (I-5) is independent of pressure, the only possibility is that the pressure dependent term on the right hand of the equation is in fact a constant. So let $\mathrm{b}=\frac{g(p) f^{\prime}(p)}{h^{\prime \prime \prime}(p)}$ where $\mathrm{b}$ is a constant. We then
get:

$$
\mathrm{i} \frac{\Omega^{2}}{k}-i k \frac{c^{2}}{\sigma}=A b
$$

After rearranging we have:

$$
\Omega^{2}-k^{2} \frac{c^{2}}{\sigma}=-i k A b
$$

This is a dispersive wave.

\section{Appendix II}

\section{A Proof of the Equality h'(p)/h"'(p) $=h(p) / h^{\prime \prime}(p)$}

The proof is fairly straightforward if one recognize the fact that the $\backslash$ propagation speed c should not be a function of height (or pressure). Since $\sigma$ is assumed independent of height, from $(7 \mathrm{c})$ :

$$
-c^{2} / \sigma=h^{\prime}(p) / h^{\prime \prime \prime}(p)
$$

Slight manipulation will give: 


$$
h^{\prime}(p)=\left(-c^{2} / \sigma\right) h^{\prime \prime \prime}(p)
$$

The equation can be solved to give:

$$
h^{\prime}(p)=A \sin \left(\frac{c}{\sqrt{\sigma}}\right)^{-1} p+B \cos \left(\frac{c}{\sqrt{\sigma}}\right)^{-1} p
$$

An integration of this equation gives:

$$
h(p)=D \cos \left(\frac{c}{\sqrt{\sigma}}\right)^{-1} p+E \sin \left(\frac{c}{\sqrt{\sigma}}\right)^{-1} p
$$

where $\mathrm{D}=\mathrm{Ac} / \sqrt{\sigma}$ and $\mathrm{E}=\mathrm{Bc} / \sqrt{\sigma}$.

The two coefficients can be determined from the two boundary conditions namely:

$$
\begin{aligned}
& \mathrm{h}=0 \text { at } \mathrm{p}=\mathrm{p}_{\mathrm{s}} \text { and } \mathrm{p}=\mathrm{p}_{\mathrm{t}} \\
& h\left(p_{s}\right)=D \cos \left(\frac{c}{\sqrt{\sigma}}\right)^{-1} p_{s}+E \sin \left(\frac{c}{\sqrt{\sigma}}\right)^{-1} p_{s}=0, \\
& h\left(p_{t}\right)=D^{\prime} \cos \left(\frac{c}{\sqrt{\sigma}}\right)^{-1} p_{t}+E^{\prime} \sin \left(\frac{c}{\sqrt{\sigma}}\right)^{-1} p_{t}=0 .
\end{aligned}
$$

There is a trivial solution $\mathrm{D}=\mathrm{E}=\mathrm{D}^{\prime}=\mathrm{E}^{\prime}=0$, but this is what we want. Another possible solution is given below:

$$
h(p)=A \sin \frac{p-p_{t}}{p_{s}-p_{t}} n \pi,
$$

where $\mathrm{n}$ is an arbitrary integer. Since this solution satisfies all the boundary conditions it must be the correct solution. Consequently,

$$
\frac{h(p)}{h^{\prime \prime}(p)}=-\frac{c^{2}}{\sigma}=\frac{h^{\prime}(p)}{h^{\prime \prime \prime}(p)} .
$$

To determine $\mathrm{n}$ let note that we have: 


$$
\begin{aligned}
h(p) & =A \sin \frac{p-p_{t}}{p_{s}-p_{t}} n \pi=A\left(\sin \frac{p n \pi}{p_{s}-p_{t}} \cos \frac{p_{t} n \pi}{p_{s}-p_{t}}-\cos \frac{p n \pi}{p_{s}-p_{t}} \sin \frac{p_{t} n \pi}{p_{s}-p_{t}}\right) \\
& =D \cos \frac{\sqrt{\sigma}}{c} p+E \sin \frac{\sqrt{\sigma}}{c} p,
\end{aligned}
$$

therefore:

$$
\frac{\sqrt{\sigma}}{c}=\frac{n \pi}{p_{s}-p_{t}} .
$$

It follows that:

$$
n=\frac{\sqrt{\sigma}\left(p_{s}-p_{t}\right)}{c \pi} .
$$

